\title{
Quantification of Ultrasound Correlation- Based Flow Velocity Mapping and Edge Velocity Gradient Measurement
}

\author{
Dae Woo Park, PhD, Grant H. Kruger, PhD, Jonathan M. Rubin, MD, PhD, James Hamilton, PhD, \\ Paul Gottschalk, PhD, Robert E. Dodde, PhD, Albert J. Shih, PhD, William F. Weitzel, MD
}

Received January 1, 2013, from the Department of Bioengineering, University of Pittsburgh, Pittsburgh, Pennsylvania USA (D.W.P.); Departments of Mechanical Engineering and Anesthesiology (G.H.K., A.J.S.), Radiology (J.M.R.), and Biomedical Engineering (R.E.D.), University of Michigan, Ann Arbor, Michigan USA; Epsilon Imaging, Inc, Ann Arbor, Michigan USA (J.R, P.G.); and Department of Internal Medicine, Veterans Administration Hospital and University of Michigan, Ann Arbor, Michigan USA (W.F.W.). Revision requested January 31, 2013. Revised manuscript accepted for publication March 4, 2013.

This work was supported in part by National Institutes of Health grant 2R44EB007842 and a grant from the Renal Research Institute. Software and imaging system support were provided by Epsilon Imaging, Inc.

Address correspondence to Dae Woo Park, PhD, Department of Bioengineering, University of Pittsburgh, 567 Scaife Hall, 3550 Terrace St, Pittsburgh, PA 15213 USA.

E-mail:bigrain@umich.edu

Abbreviations

$R F$, radiofrequency; $2 D, 2$-dimensional

doi:10.7863/ultra.32.10.1815
This study investigated the use of ultrasound speckle decorrelation- and correlationbased lateral speckle-tracking methods for transverse and longitudinal blood velocity profile measurement, respectively. By studying the blood velocity gradient at the vessel wall, vascular wall shear stress, which is important in vascular physiology as well as the pathophysiologic mechanisms of vascular diseases, can be obtained. Decorrelationbased blood velocity profile measurement transverse to the flow direction is a novel approach, which provides advantages for vascular wall shear stress measurement over longitudinal blood velocity measurement methods. Blood flow velocity profiles are obtained from measurements of frame-to-frame decorrelation. In this research, both decorrelation and lateral speckle-tracking flow estimation methods were compared with Poiseuille theory over physiologic flows ranging from 50 to $1000 \mathrm{~mm} / \mathrm{s}$. The decorrelation flow velocity measurement method demonstrated more accurate prediction of the flow velocity gradient at the wall edge than the correlation-based lateral speckle-tracking method. The novelty of this study is that speckle decorrelation-based flow velocity measurements determine the blood velocity across a vessel. In addition, speckle decorrelation-based flow velocity measurements have higher axial spatial resolution than Doppler ultrasound measurements to enable more accurate measurement of blood velocity near a vessel wall and determine the physiologically important wall shear.

Key Words_-blood flow; decorrelation; speckle tracking; ultrasound

$\mathrm{T}$ he blood flow velocity profile is important for diagnosis of cardiovascular diseases, which are associated with abnormal blood flow in arteries due to hemodynamic changes in patients. ${ }^{1}$ The blood flow velocity gradient at the wall edge has been used to estimate the vascular wall shear stress, which has a key role in vascular physiology as well as the pathophysiologic mechanisms of vascular diseases. ${ }^{2}$ Based on Poiseuille theory, the blood flow velocity profile is parabolic, and high-velocity gradients occur at the vascular wall in steady-state and fully developed laminar flow in normal conduit vessels. ${ }^{3}$ In areas with vascular tortuosity, branching, and the presence of vascular plaque, the blood flow velocity profile is asymmetric. ${ }^{1}$ Ultrasound imaging has been the preferred noninvasive technique for measuring blood flow velocity profiles.

Multigate Doppler ultrasound measurement has been applied to estimate the blood flow velocity in vessels. ${ }^{4}$ Using multigate Doppler measurements, the blood flow velocity profile and blood flow velocity gradient at the wall edge were determined from longitudinal (along the blood flow directions) views of blood vessels..$^{5-8}$ 
However, multigate Doppler ultrasound estimates of blood flow are based on 1-dimensional velocity measurements, which depend on the assumptions that the lumen is circular and that the flow is symmetric in the out-of-plane directions, both of which are violated in the presence of vascular tortuosity and branching. ${ }^{4}$

To measure the 2-dimensional (2D) flow velocity, the tracking of ultrasound radiofrequency (RF) signals also has been used to study the blood flow velocity profile. Using correlation-based lateral (across the transducer array) speckle tracking, blood flow velocity profiles have been measured from longitudinal views of blood vessels. ${ }^{9-13}$ Blood flow velocity profiles have been quantified using correlation-based lateral speckle tracking, but RF signal acquisitions on longitudinal views of blood vessels in Bmode images were limited by vascular tortuosity and branching. ${ }^{1}$ To overcome these limitations, transverse (perpendicular to the blood flow directions) views of blood vessels were used to measure blood velocity profiles. For transverse views of blood vessels, the decorrelation of ultrasound RF signals either along 1-dimensional A-lines or 2D B-mode images has been applied to the study of blood velocity profiles. Using the decorrelation of ultrasound RF signals, the vessel wall interface could be detected ${ }^{14}$ and blood flow velocity profile quantitatively identified. ${ }^{15}$ Therefore, the blood flow velocity profile measurement using the decorrelation of ultrasound RF signals from A-lines has been demonstrated.

Radiofrequency signals in B-mode images were used to determine the blood flow profiles in transverse views of a vessel. Bamber et al ${ }^{16}$ demonstrated that decorrelation could be used to identify blood flow by using the time rate of change of correlation in B-mode images. Li et al ${ }^{17}$ theoretically demonstrated a linear decrease of correlation according to elevational (out-of-plane directions) scatterer movement. Speckle decorrelation has been used to measure the motion of the transducer to produce 3-dimensional ultrasound images. ${ }^{17-20}$ This speckle decorrelation measurement has been applied by Rubin et $\mathrm{al}^{21}$ to qualitatively identify the blood flow profiles in both in vitro flow phantom and in vivo animal studies. Rubin et $\mathrm{al}^{22}$ also used Doppler flow measurements to improve the accuracy of volumetric flow from the decorrelation-based flow profiles and theoretically model an exponential decrease in correlation according to scatterer movement. Bamber et al, ${ }^{16}$ Li et al, ${ }^{17}$ and Rubin et $\mathrm{al}^{21,22}$ demonstrated that speckle decorrelation could generate flow velocity profiles, but the decorrelation was not experimentally measured as a function of the position along the beam, and the flow velocity profile was not quantified.
The first goal of this research was to quantitatively estimate flow profiles using speckle decorrelation. The novelty of this study is that speckle decorrelation-based flow velocity measurements determine blood velocity profile across a vessel and enable entire blood velocity measurements along the vessel. In addition, speckle decorrelationbased flow velocity measurements can measure blood velocities near the vessel wall with high spatial resolution, allowing determination of physiologically important wall shear. In contrast, Doppler ultrasound measurements lack the spatial resolution to determine blood velocity near a vessel wall.

Speckle decorrelation can be used to measure velocity profiles because as blood particles travel through an ultrasound imaging plane, the received echo signals decorrelate at a rate that is related to the flow velocity. ${ }^{23-26}$ To achieve this goal, a calibration experiment was performed. The transducer scan plane was moved elevationally with a series of constant velocities across a tissue-mimicking phantom. The relationship between the transducer elevational velocity and the frame-to-frame decorrelation as a function of position along beam was obtained. This calibration could then be used to calculate velocity from a $2 \mathrm{D}$ transverse image with a specifically observed decorrelation.

In this study, the wall edge detection estimation was proposed to obtain the velocity gradients at the wall edges. The wall edge was estimated using both B-mode images and second-order gradients of flow velocity profiles at the wall edge. The entire vascular wall edges can be determined using this edge detection estimation. The accuracy of the ultrasound-measured flow velocity profile and the velocity gradient was evaluated by comparison with a parabolic velocity profile based on Poiseuille theory.

The second goal of this research was to compare the transverse decorrelation-based and lateral speckle-tracking flow velocity profiles. For lateral speckle-tracking flow velocity measurement, ultrasound RF signals were acquired from longitudinal views of a lumen in a flow phantom through which blood-mimicking fluid was flowing. The wall edge was also determined using both B-mode images and second-order gradients of the flow velocity profiles. Velocity gradients were measured at the wall edge from the flow velocity profiles. The flow velocity profiles and the velocity gradients were compared with the decorrelation flow velocity profiles and velocity gradients.

\section{Materials and Methods}

To obtain the data required to achieve the 3 goals, 2 experiments were performed: (1) speckle decorrelation meas- 
urement using a tissue-mimicking phantom with a motion control system; and (2) in vitro flow measurement using blood-mimicking fluid with a flow phantom. Two methods, decorrelation-based transverse velocity estimation and lateral speckle-tracking velocity estimation, were used to measure the flow velocity profile and the velocity gradient at the wall edge. For the decorrelation-based velocity estimation, the experimental setup shown in Figure 1A was used to obtain calibration data, and the setup shown in Figure 2 was used to obtain the flow data.

The first experimental setup for speckle decorrelation measurement, as shown in Figure 1A, consists of a tissuemimicking phantom, a stepper motor-driven motion control system, and an ultrasound transducer. The tissue-

Figure 1. A, Linear rail system and tissue-mimicking phantom. B, Definition of directions, beam correlation width, elevational transducer velocity, and speckle decorrelation in the phantom.

A

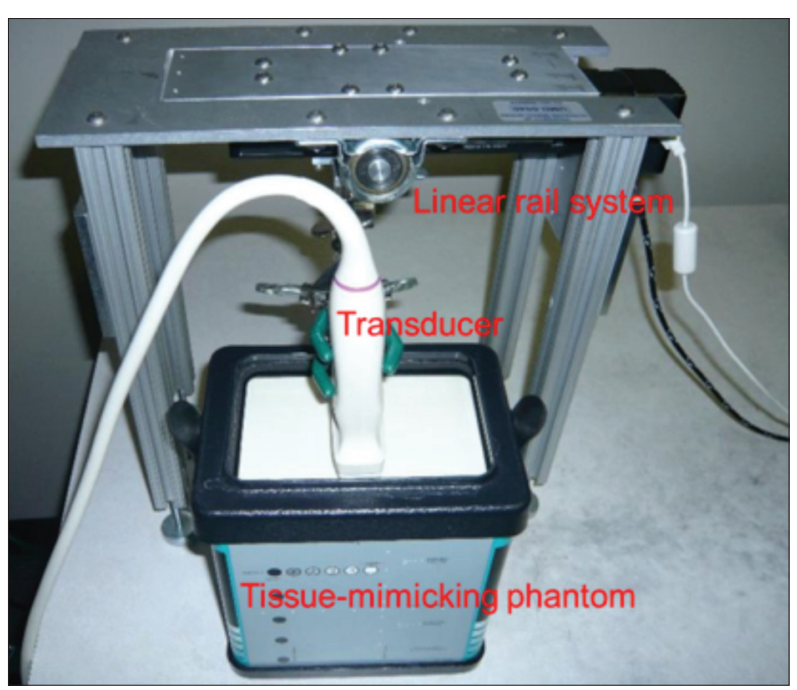

B $x$ : Elevational direction $\quad B_{x}$ : Beam correlation width $y$ : Lateral direction $\quad V_{x}$ : Elevational transducer velocity $z$ : Axial direction $\quad D:$ Speckle decorrelation

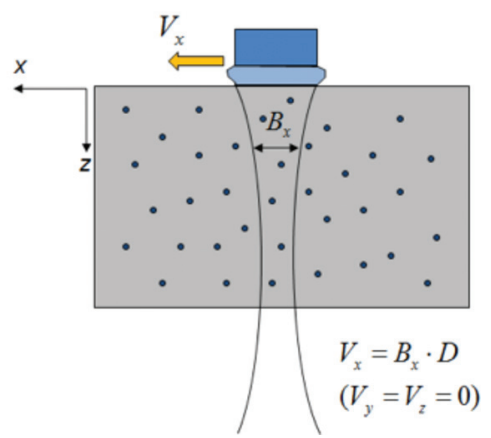

mimicking phantom contains scatterers producing fully developed speckle. The motion control system (Epsilon Imaging, Inc, Ann Arbor, MI) moves the transducer over the surface of a tissue-mimicking phantom, resulting in the formation of a series of position-dependent speckle patterns as each frame is generated.

The ultrasound scanner (DCI 6000; Epsilon Imaging, Inc) with a 9-MHz linear array acquires a series of $19.5 \times$ 24.9-mm $(215 \times 278$-pixel $)$ beam-formed RF frames, consisting of speckle spots of $0.45 \times 0.27 \mathrm{~mm}(5 \times 3$ pixels $)$, with incremental spacing in the elevational direction over a tissue-mimicking phantom. A $7 \times 8$ grid is chosen in the RF frame, with each grid element defining a region of interest. Each region of interest is $2.7 \times 2.7 \mathrm{~mm}(30 \times 30$ pixels $)$, which is adequate for noise reduction to produce consistent correlation values, and the region of interest has sufficient speckle spots to perform the speckle decorrelation. EchoInsight software (Epsilon Imaging, Inc) executes $2 \mathrm{D}$ speckle tracking using a $0.45 \times 0.27-\mathrm{mm}(5 \times 3$-pixel $)$ kernel, a $0.81 \times 0.45-\mathrm{mm}(9 \times 5$-pixel $)$ filter, and a finite impulse response high-pass filter for compounding the RF signals. The finite impulse response high-pass temporal filtering suppresses reverberation noise, enhances the RF signal decorrelation between frames, hence improves the accuracy of wall edge detection.

Figure 1B shows the beam correlation width, elevational transducer velocity, and speckle decorrelation in the phantom speckle decorrelation measurement. The beam correlation width is defined as the transducer movement that reduces the correlation value to half of the full correlation range (ie, maximum-minimum correlation), as illustrated in Figure $3 \mathrm{~A}^{27}$ The beam correlation width is related to the maximum flow velocity that can be measured. When

Figure 2. Graduated cylinder, peristaltic pump, and flow phantom.

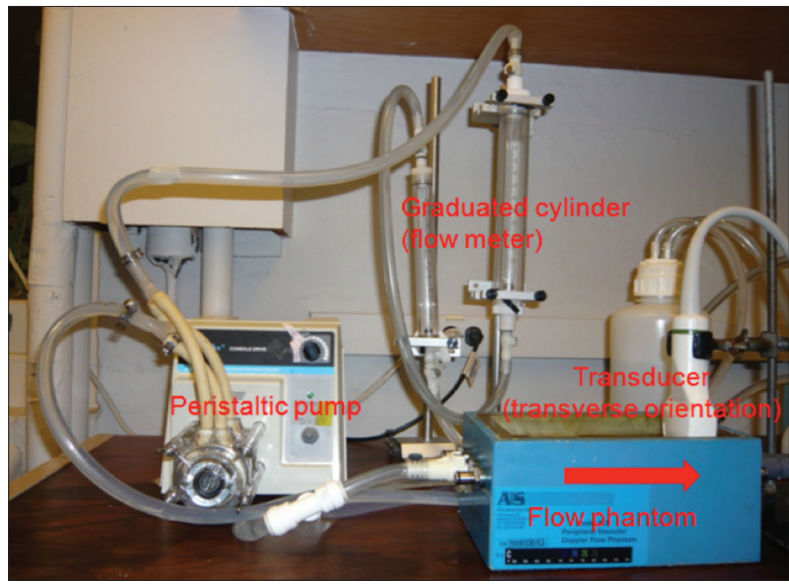


the transducer movement during the time between two consecutive firings of the transducer exceeds the beam correlation width, complete loss of speckle tracking occurs, ${ }^{22}$ which limits the maximum flow velocity that can be measured using the decorrelation-based method and will be evident later in the "Results" and "Discussion" sections. In addition, the beam correlation width versus depth represents the ultrasound beam profile, which can qualitatively validate the proposed method.

For the first experimental setup (Figure 1A), $47 \mathrm{RF}$ frames were acquired over the motion steps of the transducer. The step distance was $0.044 \mathrm{~mm}$ with a 0.1 -second time between steps. Two-dimensional speckle motion tracking ${ }^{21,22}$ was performed for the RF frames using the EchoInsight software by increasing the lag from 1 to 46 . Lag can be defined as the number of frames skipped between the frames to which tracking is applied. The mean and standard error of the scatterer movement versus correlation were obtained for each depth layer of the $7 \times 8$ grid from the RF frames.

A

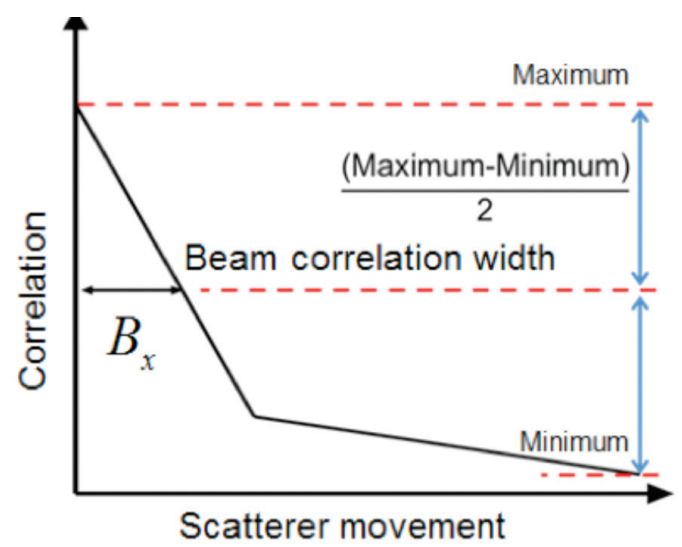

B

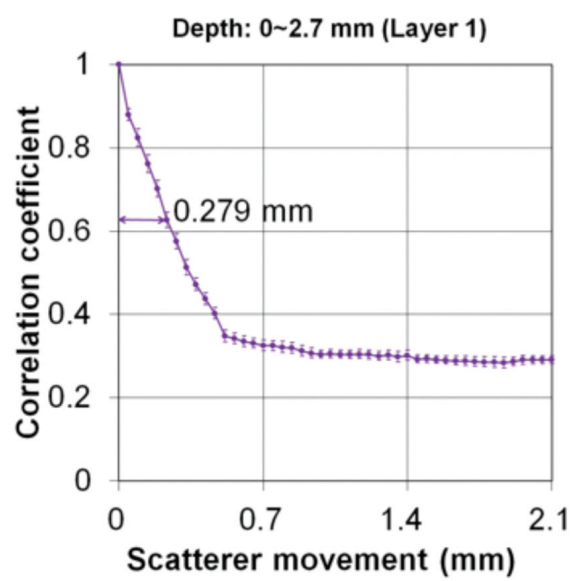

The second experimental setup for in vitro flow measurement consists of a peristaltic pump, a graduated cylinder, a flow phantom, and an ultrasound transducer, as shown in Figure 2. The peristaltic pump (Barnant, Barrington, $\mathrm{IL}$ ) circulates a blood-mimicking fluid mixture. The fluid consists of water and glycerin in a ratio of 5:1 with plastic particles of $30 \pm 3 \mu \mathrm{m}$ diameter at a concentration of $1.7 \times 10^{4}$ particles $/ \mathrm{mL}$ (ATS Laboratories, Bridgeport, CT) to increase the backscatter signal. Mean volume flow rates of $50,100,150$, and $200 \mathrm{~mL} / \mathrm{min}$, within the range of human blood flow rates between 1 and $1000 \mathrm{~mL} / \mathrm{min},{ }^{28}$ were studied. The mean volume flow rate was measured using a graduated cylinder flow meter. The flow phantom (model 525; ATS Laboratories), made of tissue-mimicking urethane rubber, has a $5.5-\mathrm{mm}$ diameter lumen as the flow path.

In the second experimental setup (Figure 2), $700 \mathrm{RF}$ frames were acquired at an acoustic frame rate of $348 \mathrm{~Hz}$ from the transverse view of the flow phantom lumen.

Figure 3. A, Anticipated scatterer movement versus correlation curve and beam correlation width of the curve. B, Means and standard errors of scatterer movement-versus-correlation curves from 8 regions of interest in 2 layers, each measuring $2.7 \mathrm{~mm}$ in height.

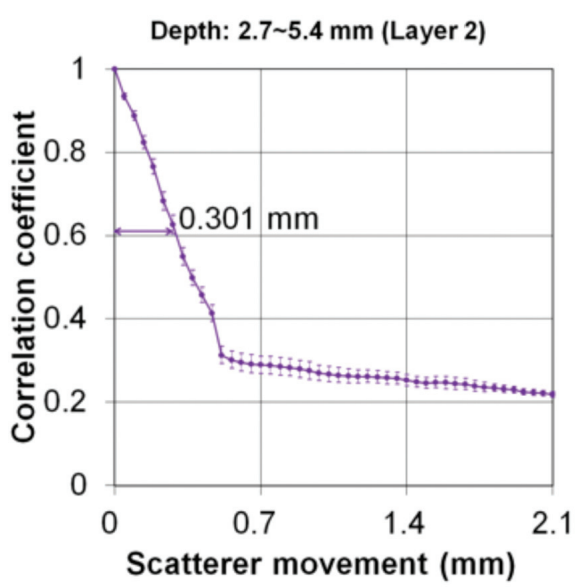


The correlation map was computed as a function of time $^{21,22}$ using the EchoInsight software. The correlation map of the flow phantom was used to determine the decorrelation rate corresponding to the fluid motion. The decorrelation of the fluid mixture and the mean scatterer movement-versus-correlation curves determined from the first experiment were used to measure the scatterer displacement. The scatterer displacement in two consecutive frames was multiplied by the frame rate to determine the flow velocity. Using an averaged correlation map from the complete 700 -frame set, the flow velocity profile was obtained for the cross section of the lumen. This flow velocity profile was used to determine the velocity gradient at the wall edge using linear regression of the velocity curve.

For lateral speckle-tracking velocity estimation, ${ }^{13}$ the second experimental setup (Figure 2) was used to measure the flow velocity from the longitudinal view of the flow phantom. Radiofrequency images with 917 frames were acquired with a $426-\mathrm{Hz}$ frame rate to obtain the lateral velocity map of the fluid mixture flowing at a specific velocity. Using an averaged lateral velocity map from the entire range of 917 frames, the flow velocity profile was obtained for the longitudinal cross section of the lumen. Similarly, the velocity gradient at the wall edge was determined from this flow velocity profile using a linear regression estimate assuming that the flow velocity is zero at the wall.

The wall edges were determined using the B-mode image and the second-order spatial derivative of the flow velocity profile. In the B-mode image, pixels in the vicinity of wall edges were identified from the brightness change of grayscale pixels due to the acoustic impedance of the scatterer density change between the tissue-mimicking phantom and the fluid mixture. ${ }^{29}$ Among these pixels, the wall edges were determined by the highest value of the second-order gradient of the velocity profile.

\section{Results}

\section{Decorrelation-Based Flow Velocity Profile Measurement and Edge Detection}

Figure $3 \mathrm{~B}$ shows the mean and standard error of the scatterer movement-versus-correlation curves in the top 2 layers (depth, $0-5.4 \mathrm{~mm}$ ). The correlation coefficient and the scatterer movement represent a normalized covariance and the elevational displacement of scatterers through the beam, respectively. The depth is defined from the top surface of the phantom with the transducer in the position for the transverse view. The correlation coefficients and scatterer movements in all 7 layers are summarized in Table 1. The sharp and linear decrease in the correlation coefficient from 1.0 to
0.3 was observed after about $0.5 \mathrm{~mm}$ of speckle movement in the 7 layers. The linear decrease in the correlation coefficient versus scatterer movement was proposed by $\mathrm{Li}$ et al ${ }^{17}$ and observed in all layers. The beam correlation width was approximately $0.30 \mathrm{~mm}$ in the top 4 layers (depth, 0-10.9 $\mathrm{mm}$ ) and steadily increased to $0.36 \mathrm{~mm}$ in deeper layers (depth, 10.9-19.0 mm). The divergence of the beam correlation width due to phased array focusing was observed.

The beam correlation width measurement was repeated 4 times. Figure 4 shows the beam correlation width measurements versus depth in the 7 layers. Layers 2,3 , and 4 had consistent beam correlation width measurements with low standard errors (0.003-0.005 mm). The ultrasound beam then gradually diverged from layers 5 to 7 . This divergence of beam correlation width versus depth was proposed by Shung ${ }^{30}$ and qualitatively validated the measurement and analysis. The beam width was theoretically calculated from Equation 1 and compared with the average beam correlation width. The beam width is defined as full width at half maximum (FWHM) corresponding to the $-6-\mathrm{dB}$ points ${ }^{31}$ :

$$
\mathrm{FWHM}=1.03 \lambda F / D,
$$

where $\lambda$ is the wave length, $F$ is the focused depth, and $D$ is the transducer diameter. The beam width was about 0.3 $\mathrm{mm}$ at a $13-\mathrm{mm}$ depth, $8-\mathrm{mm}$ elevational aperture size, and

Table 1. Correlation Coefficients and Scatterer Movements in 7 Layers, Each Measuring $2.7 \mathrm{~mm}$ in Height

\begin{tabular}{cccc}
\hline Depth & $\begin{array}{c}\text { Beam } \\
\text { Width, } \mathbf{m m}\end{array}$ & $\begin{array}{c}\text { Speckle Scatterer } \\
\text { Movement, } \mathbf{~ m m}\end{array}$ & $\begin{array}{c}\text { Correlation } \\
\text { Coefficient }\end{array}$ \\
\hline Layer 1 & 0.279 & 0.0 & 1.00 \\
& & 0.5 & 0.34 \\
Layer 2 & 0.301 & 2.1 & 0.29 \\
& & 0.0 & 1.00 \\
Layer 3 & 0.311 & 0.5 & 0.31 \\
& & 2.1 & 0.22 \\
Layer 4 & 0.307 & 0.0 & 1.00 \\
& & 0.5 & 0.31 \\
Layer 5 & 0.331 & 0.0 & 0.18 \\
& & 0.5 & 1.00 \\
& & 2.1 & 0.29 \\
Layer 6 & 0.344 & 0.0 & 0.19 \\
& & 0.5 & 1.00 \\
& & 2.1 & 0.27 \\
Layer 7 & 0.345 & 0.0 & 0.20 \\
& & 0.5 & 1.00 \\
& & 2.1 & 0.29 \\
& & 0.0 & 0.19 \\
& & 0.5 & 1.00 \\
& & 2.1 & 0.37 \\
& & & 0.19 \\
\hline
\end{tabular}


9-MHz center frequency. This beam width was similar to the average beam correlation width $(\approx 0.3 \mathrm{~mm})$ in layer 5 .

Scatterer movement-versus-correlation curves were applied to quantify the flow velocity using $2 \mathrm{D}$ speckle tracking of the flow phantom with the fluid mixture flowing at a mean volume flow rate of $100 \mathrm{~mL} / \mathrm{min}$ using the second experimental setup (Figure 2). Figure 5A shows the B-mode image of the transverse view of the lumen with the fluid mixture in the flow phantom. A total of 700 consecutive RF frames were acquired in 2.0 seconds. Two-dimensional motion estimation was performed for the 700 frames by correlating adjacent frames (with a lag of 1) to generate 699 correlation maps. In each pixel, the correlation coefficient in these 699 correlation maps was averaged to obtain the averaged correlation map as shown in Figure 5B.

A line, denoted $a-a$, was selected across the center of the lumen in the averaged correlation map (Figure 5B). The correlation coefficient of the pixels along the a-a line was obtained 5 times under the same $(100-\mathrm{mL} / \mathrm{min})$ volume flow rate to observe the variation of the correlation profiles between measurements. The means and standard errors of the correlation coefficients along the a-a line are shown in Figure 6 for the mean flow rates of 100 as well as 50,150 , and $200 \mathrm{~mL} / \mathrm{min}$; each also had 5 measurements using the RF frames of the transverse view of the lumen in the flow phantom. The correlation coefficient of pixels in the a-a line was approximately 1.0 up to about $6.6 \mathrm{~mm}$, the depth of the top edge of the lumen. Inside the lumen, the correlation coefficient gradually dropped to a minimum value in the middle of the lumen where the flow velocity

Figure 4. Beam correlation width versus depth in 7 layers, each measuring $2.7 \mathrm{~mm}$ in height. Circles indicate measurement 1; triangles, measurement 2; squares, measurement 3; and diamonds, measurement 4.

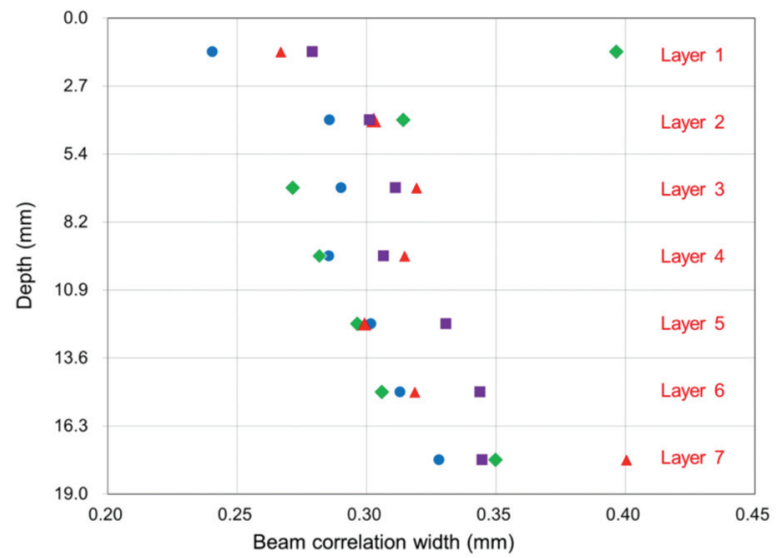

was the highest and then increased back to 1.0 at the bottom edge of the lumen. The change in the correlation coefficient across the lumen demonstrated the feasibility of using the correlation coefficient to estimate the flow velocity profile, as proposed by Rubin et al. ${ }^{21}$ The minimum mean correlation coefficients for mean volume flow rates of $50,100,150$, and $200 \mathrm{~mL} / \mathrm{min}$ were $0.92,0.72$, 0.65 , and 0.56 , respectively. A high flow rate generated a lower minimum mean correlation coefficient, which was also observed by Rubin et al. ${ }^{21}$

To quantify the flow velocity using the correlation coefficient, the depth along the a-a line was divided into 7 layers, each measuring $2.7 \mathrm{~mm}$ in height, similar to the speckle decorrelation measurement. These 7 layers are shown in Figure 6. In each layer, the value of the correlation

Figure 5. B-mode image (A) and correlation map (B) of the transverse view of the lumen with the fluid mixture flowing at the mean volume flow rate of $100 \mathrm{~mL} / \mathrm{min}$ in the flow phantom.

A

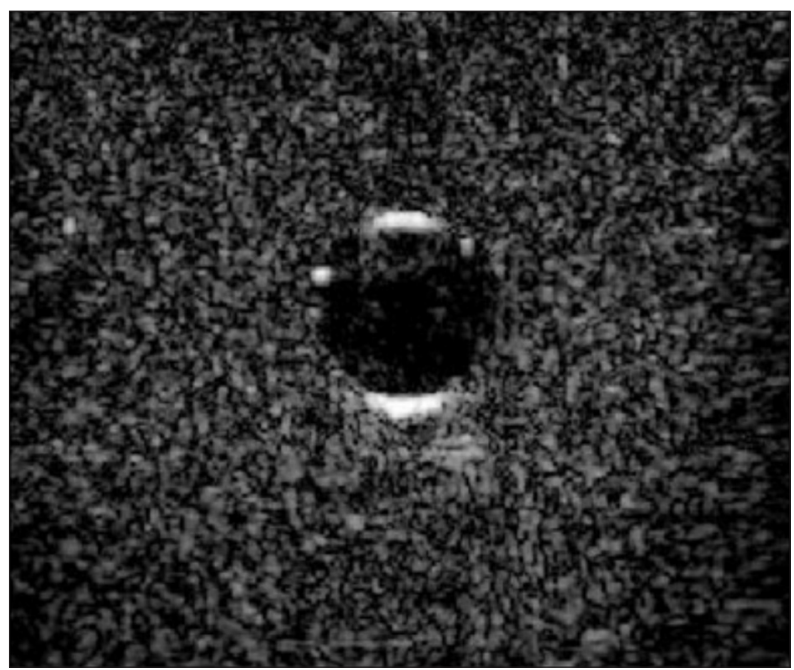

B

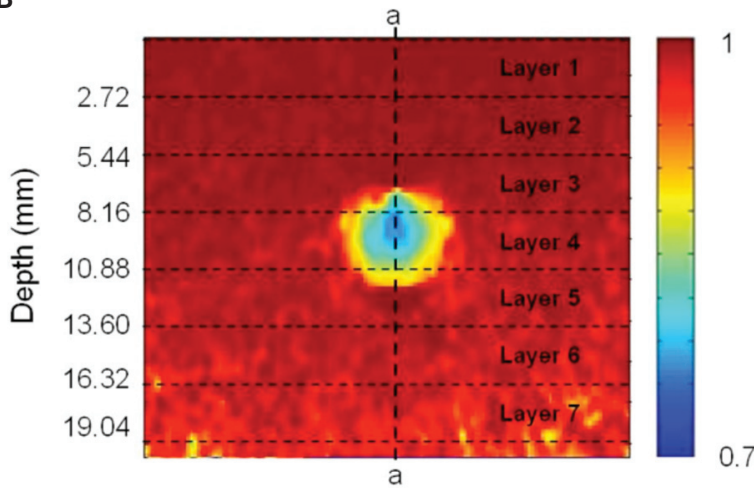


coefficient along the a-a line was used to find the amount of scatterer movement in two consecutive frames based on the mean scatterer movement-versus-correlation curves in Figure 3B. The scatterer movement in the fluid mixture was determined from layers 3,4 , and 5 : the location of the lumen in the flow phantom. The scatterer movement in two consecutive frames was multiplied by the acoustic frame rate $(348 \mathrm{~Hz})$ to estimate the flow velocity.

The open squares in Figure 7 show the velocity profile estimated using the decorrelation-based flow velocity measurement method at mean volume flow rates of 50, 100,150 , and $200 \mathrm{~mL} / \mathrm{min}$. The theoretical (parabolic) velocity profiles, which are represented by solid lines, are also presented for comparison. Near the top and bottom edges, the sharp increase in the flow velocity was the same for both the decorrelation-based flow velocity profile and the theoretical velocity profile. This result demonstrated the feasibility of the decorrelation-based flow velocity measurement method for estimating the flow velocity gradient at the edge (not the whole velocity profile) of the lumen. In the middle of the lumen, the decorrelation-based flow velocity measurement underestimated the flow velocity because the scatterer movement during the time $(0.003$ seconds) between two consecutive firings of the transducer was greater than the beam correlation width. This phenomenon has been observed by Rubin et al. ${ }^{22}$

The top and bottom wall edges were determined using the edge detection method based on the secondorder gradient of the velocity profile. Figure $8 \mathrm{~A}$ shows the B-mode image of the transverse view of the lumen with the fluid mixture flowing at the mean volume flow rate of 100 $\mathrm{mL} / \mathrm{min}$. The a-a line across the top and bottom edges and

Figure 6. Means and standard errors of the depth-versus-correlation coefficient curves from line a-a for mean volume flow rates of 50,100, 150, and $200 \mathrm{~mL} / \mathrm{min}$.

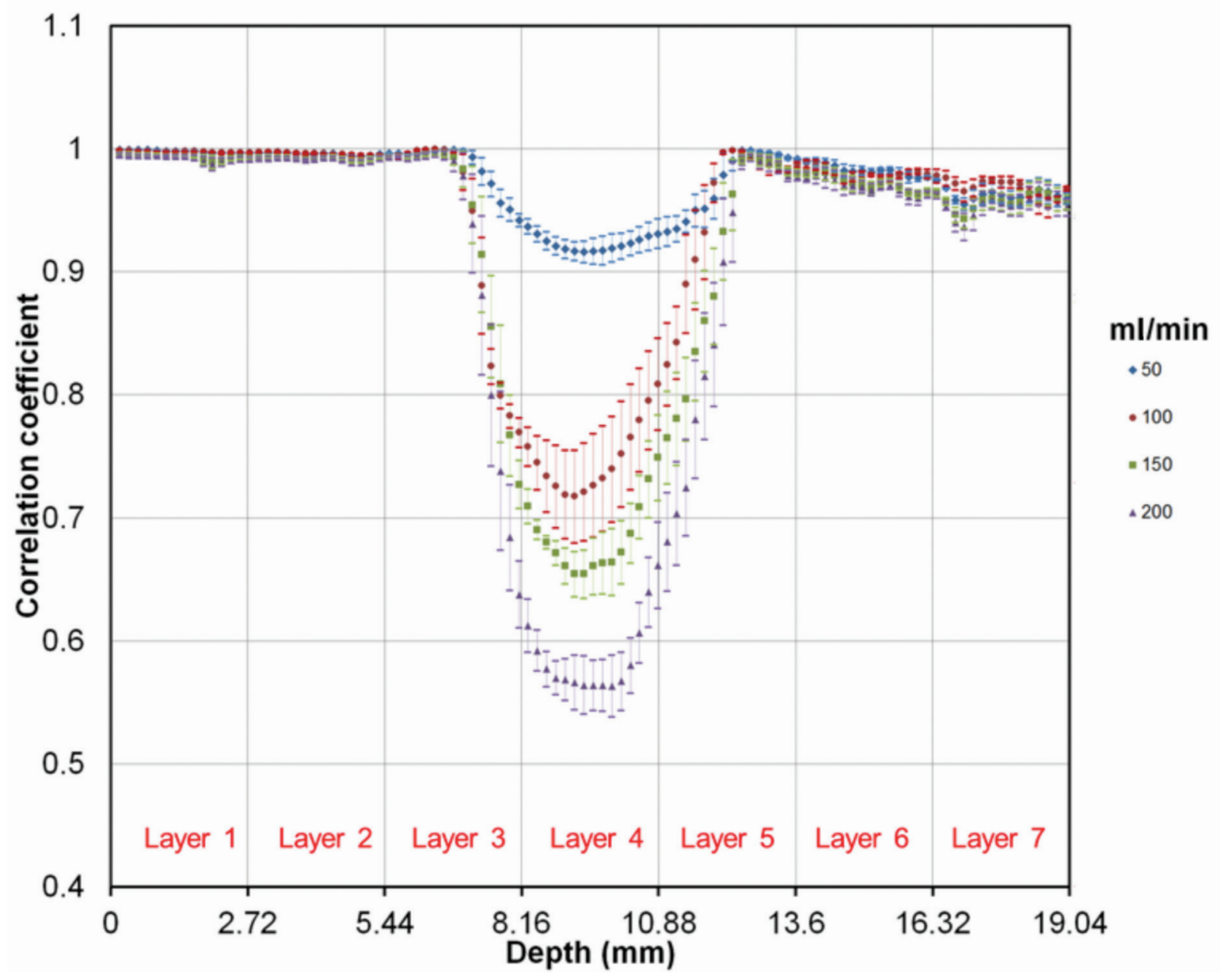


center of the lumen is marked. Close-up views of pixels in the lumen and near the top and bottom edges as well as the a-a line are shown in Figure 8B. Four pixels near the top edge, denoted $\mathrm{T} 1, \mathrm{~T} 2, \mathrm{~T} 3$, and $\mathrm{T} 4$, were identified based on the transition of the gray scale at depths of $6.45,6.63$, 6.82 , and $7.00 \mathrm{~mm}$, respectively. The second-order gradient at $\mathrm{T} 1, \mathrm{~T} 2, \mathrm{~T} 3$, and $\mathrm{T} 4$ and adjacent pixels along the a-a line was determined and is shown in Figure 9A for the mean volume flow rate of $100 \mathrm{~mL} / \mathrm{min}$. Pixel T3 had the highest second-order gradient $(243 \mathrm{~mm} / \mathrm{s})$ among the 4 pixels and was determined as the top edge. It is noted that pixels around $\mathrm{T} 1$ and $\mathrm{T} 4$ could have higher second-order gradient values but not be considered as the wall edge because they are not in the group of pixels originally selected.
Similarly, 4 pixels, denoted B1, B2, B3, and B4 in Figure $8 \mathrm{~B}$, were selected near the bottom edge at depths from 12.16 to $12.71 \mathrm{~mm}$. The second-order gradient at B1, B2, $\mathrm{B} 3$, and $\mathrm{B} 4$ and adjacent pixels along the a-a line was determined and is shown in Figure 9A for the mean volume flow rate of $100 \mathrm{~mL} / \mathrm{min}$. Pixel B1 had the highest second-order gradient $(280 \mathrm{~mm} / \mathrm{s})$ among the 4 pixels and was determined as the bottom edge. This edge detection method was repeated for mean volume flow rates of 50,150, and $200 \mathrm{~mL} / \mathrm{min}$ to obtain the top and bottom wall edges.

Figure 7. Flow velocity profiles across a center of a cross section of the lumen in the flow phantom at mean volume flow rates of 50, 100, 150, and $200 \mathrm{~mL} / \mathrm{min}$. The correlation-based lateral speckle-tracking velocity profile was translated to depths from 6 to $13 \mathrm{~mm}$ for comparison. Open circles indicate lateral speckle-tracking velocity profile; open squares, decorrelation transverse velocity profile; solid purple lines, upper limit of decorrelation velocity; and solid red lines, theoretical (parabolic) velocity profile.
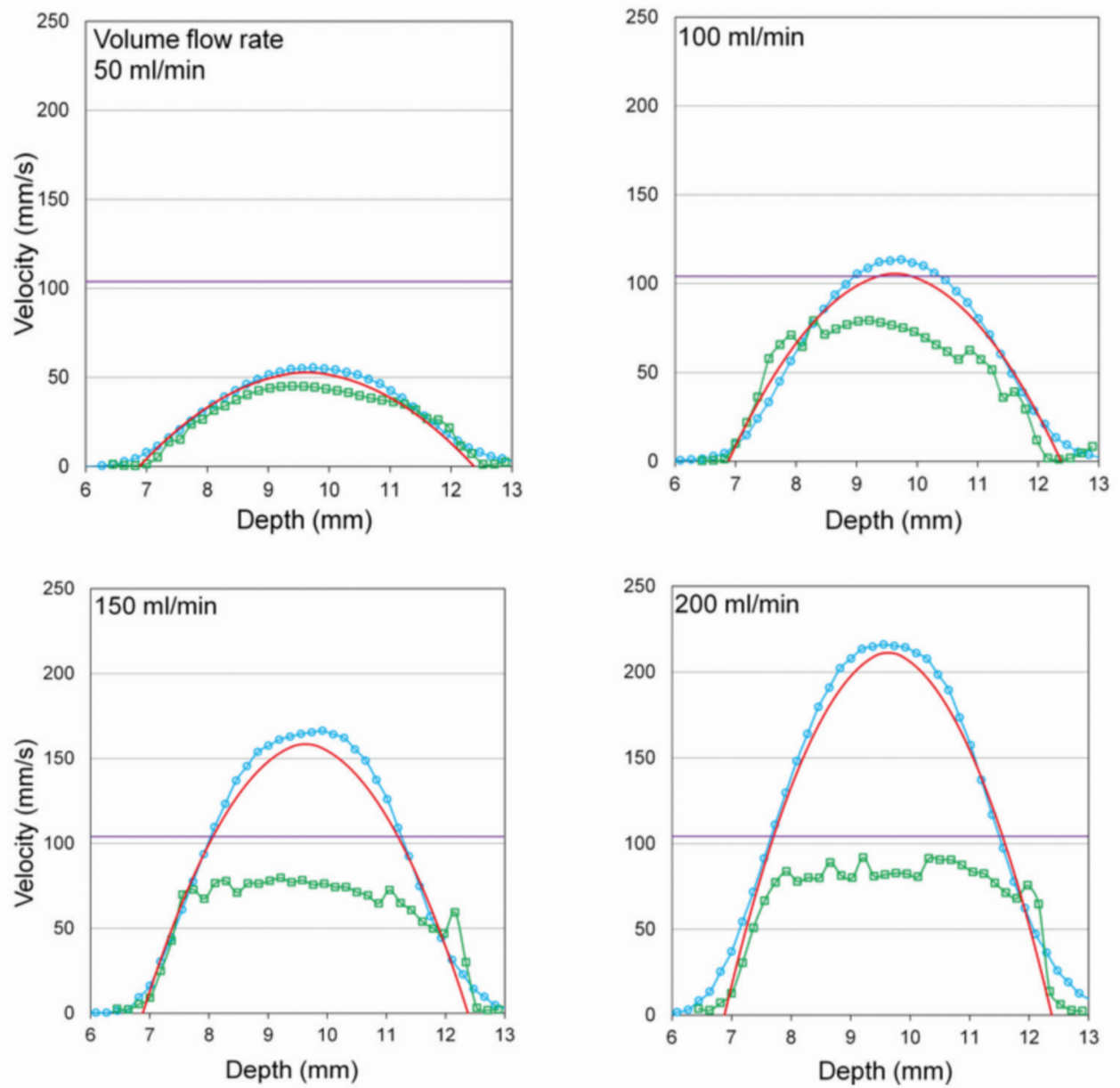


\section{Lateral Speckle-Tracking Flow Velocity Profile Measurement and Edge Detection}

The flow velocity profile was also measured using correlationbased lateral speckle tracking from the flow phantom. A fluid mixture was used that flowed at mean volume flow rates of $50,100,150$, and $200 \mathrm{~mL} / \mathrm{min}$ in the second experimental setup (Figure 2). Figure 10A shows the B-mode image of the longitudinal view of the lumen with the mean volume flow rate of $150 \mathrm{~mL} / \mathrm{min}$ in the flow phantom. A total of 917 consecutive RF frames (in 2.15 seconds) were acquired. Two-dimensional speckle tracking was performed by correlating adjacent frames (1-frame lag) to generate 916 lateral velocity maps. In each pixel, the lateral velocity values in these 916 lateral velocity maps were aver- aged to obtain the averaged lateral velocity map, as shown in Figure $10 \mathrm{~B}$ for the mean flow rate of $150 \mathrm{~mL} / \mathrm{min}$. Due to the pulsatile flow from the peristaltic pump (Figure 2), the flow velocity was not consistent along the lumen, as shown in Figure 10B. The 274 transducer beams were selected across the lumen from the entire B-mode image to measure all the flow velocity profiles in the flow phantom. The means and standard deviations of flow velocities were calculated from the 274 flow velocity profiles. The standard deviations of flow velocity profiles were between 0.5 and $2 \mathrm{~mm} / \mathrm{s}$ and confirmed that the average flow velocity profile represented well the entire flow velocity profiles in the flow phantom. Ten lines spanned by 30 pixels, as shown in Figure 10B, were selected across the lumen. The total flow rate of the flow velocity profile measured using

Figure 8. A, B-mode image of the transverse view of the lumen with the fluid mixture flowing at the mean volume flow rate of $100 \mathrm{~mL} / \mathrm{min}$. B, Closeup views of pixels in the lumen and near the top and bottom edges as well as the a-a line.

A
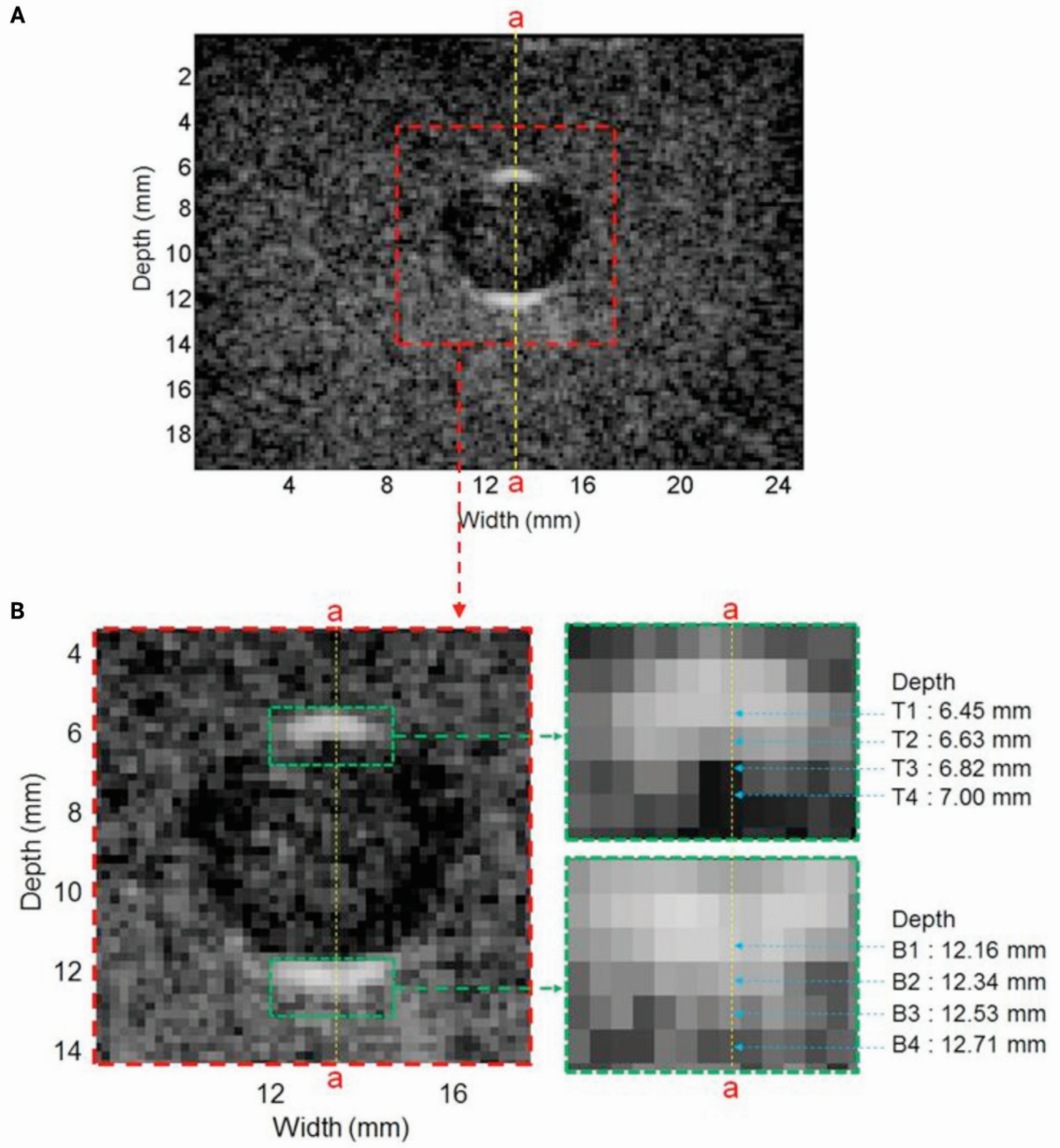
lateral speckle tracking in each line was calculated. The line with the flow rate closest to $150 \mathrm{~mL} / \mathrm{min}$, denoted as b-b in Figure 10B, was selected to represent the flow velocity profile at the mean volume flow rate of $150 \mathrm{~mL} / \mathrm{min}$. We desired to determine the velocity gradient from any individual lines, and the flow velocity profile from the b-b line was selected for the velocity gradient analysis. This procedure was repeated for mean flow rates of 50,100, and $200 \mathrm{~mL} / \mathrm{min}$ to find the cross-sectional line that had the integrated volume flow rate best matched to the target flow rate.

The flow velocity profiles estimated using the lateral speckle-tracking flow velocity measurement method at mean volume flow rates of $50,100,150$, and $200 \mathrm{~mL} / \mathrm{min}$ are shown by open circles in Figure 7. The lateral speckletracking flow velocity measurement method spread beyond both edges due to the correlation window used that had a $0.54 \times 0.81-\mathrm{mm}$ size, which caused the spatial smoothing effect of the flow velocity profile at the edge of the vessel. ${ }^{32}$

The top and bottom wall edges were also determined using the edge detection method based on the secondorder gradient of the velocity profile. Figure $11 \mathrm{~A}$ shows the B-mode image of the longitudinal view of a lumen with the fluid mixture flowing at the mean volume flow rate of 150 $\mathrm{mL} / \mathrm{min}$. For the lateral speckle tracking (longitudinal view), the depth of the lumen in the flow phantom was 6.9 $\mathrm{mm}$ deeper than that in the decorrelation-based flow velocity measurement (transverse view shown in Figure 5) because the surface of the flow phantom has a rectangular $10 \times 5$-cm indentation, $6.9 \mathrm{~mm}$ deep, which was used as a platform for transverse view but not longitudinal view measurements. The depth at which the lateral speckle

Figure 9. A, Flow velocity profiles and second-order gradient of the flow velocity profile across the a-a line from speckle decorrelation flow measurements at the mean volume flow rate of $100 \mathrm{~mL} / \mathrm{min}$. B, Flow velocity profiles and second order-gradient of the flow velocity profile across the $b$ $\mathrm{b}$ line from lateral speckle-tracking measurements at the mean volume flow rate of $150 \mathrm{~mL} / \mathrm{min}$. Downward arrows indicate bottom edges; and upward arrows, top edges.

A
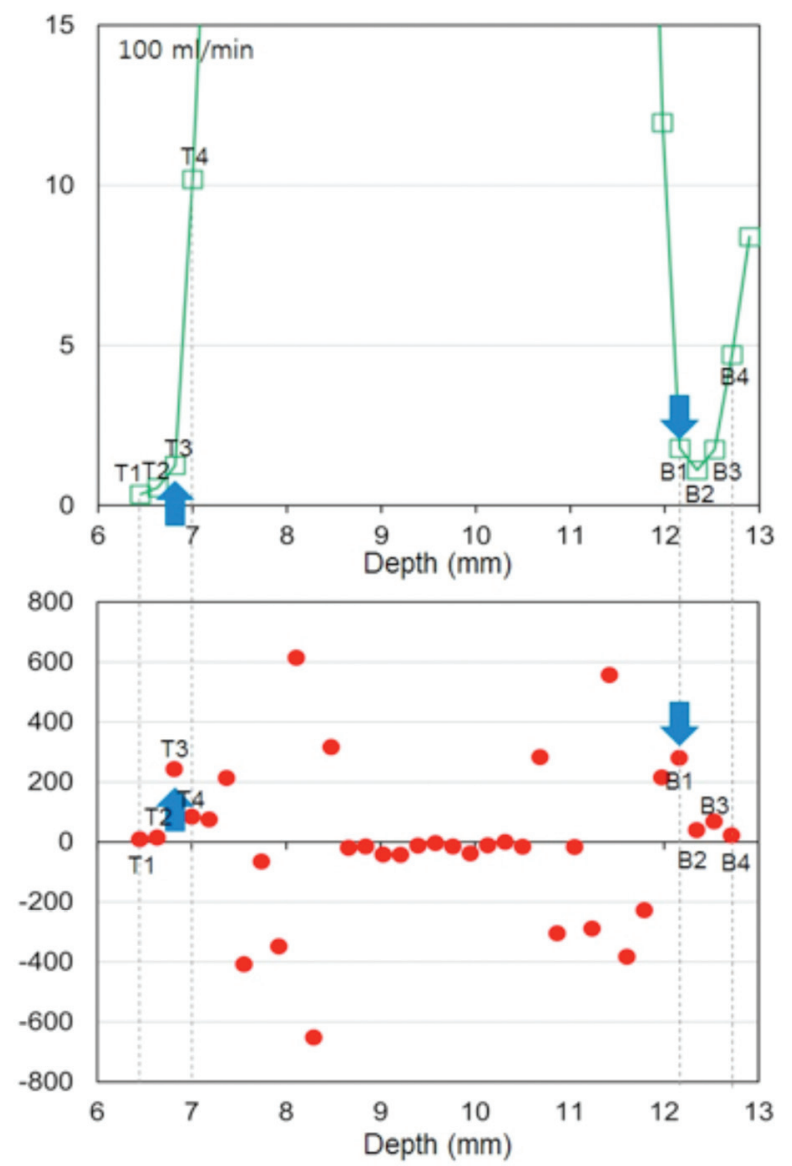

B
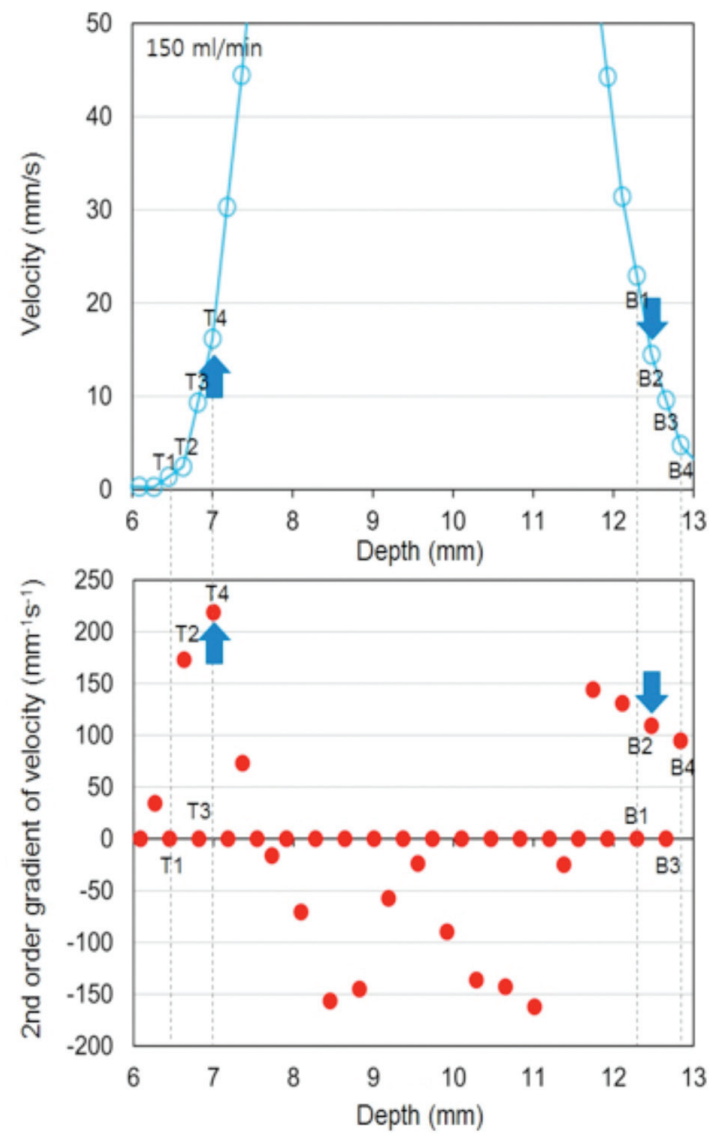
tracking was performed was offset by this value for mutual comparison of the measurement results using these two setups. The $b-b$ line with the integrated volume flow rate closest to $150 \mathrm{~mL} / \mathrm{min}$ is marked. Close-up views of pixels in the b-b line near the lumen are shown in Figure 11B. Four pixels near the top edge, denoted T1, T2, T3, and T4, were identified at depths of $6.45,6.63,6.82$, and $7.00 \mathrm{~mm}$, respectively. The second-order gradient at $\mathrm{T} 1, \mathrm{~T} 2, \mathrm{~T} 3$, and $\mathrm{T} 4$ and adjacent pixels along the $\mathrm{b}-\mathrm{b}$ line was determined and is shown in Figure 9B for the mean volume flow rate of $150 \mathrm{~mL} / \mathrm{min}$. Pixel T4 had the highest second-order gradient $(219 \mathrm{~mm} / \mathrm{s})$ among the 4 pixels and was determined as the top edge.

Figure 10. B-mode image (A) and lateral velocity map (B) of the longitudinal view of the lumen with the fluid mixture flowing at the mean volume flow rate of $150 \mathrm{~mL} / \mathrm{min}$ in the flow phantom.

A

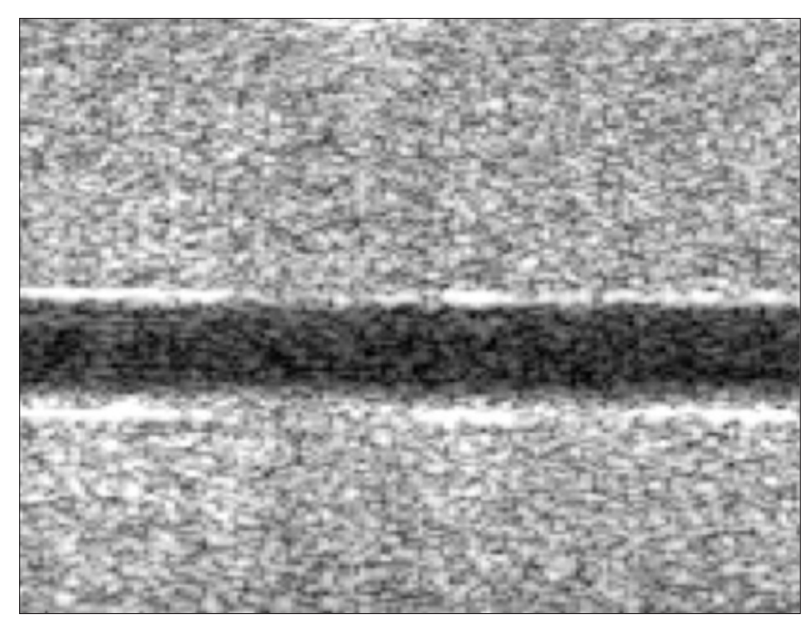

B

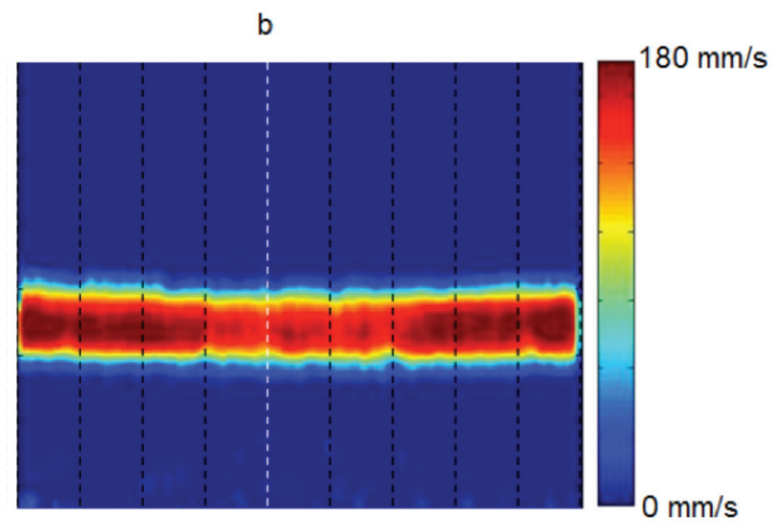

b
Similarly, 4 pixels, denoted B1, B2, B3, and B4 in Figure $11 \mathrm{~B}$, were selected near the bottom edge at depths of $12.29,12.47,12.66$, and $12.84 \mathrm{~mm}$. The second-order gradient at B1, B2, B3, and B4 and adjacent pixels along the b$\mathrm{b}$ line was determined and is shown in Figure $9 \mathrm{~B}$ for the mean volume flow rate of $150 \mathrm{~mL} / \mathrm{min}$. Pixel B2 had the highest second-order gradient $(109 \mathrm{~mm} / \mathrm{s})$ among the 4 pixels and was determined as the bottom edge. This edge detection method was repeated for mean volume flow rates of 50,100 , and $200 \mathrm{~mL} / \mathrm{min}$ to obtain the top and bottom wall edges. The diameter subtracted between the top and bottom edges was $5.84 \mathrm{~mm}$, and this value was close to the 5.5-mm-diameter lumen of the flow phantom.

\section{Velocity Gradient Estimation}

The velocity gradient at the wall edge was estimated using the linear regression of the flow velocity at 3,4 , and 5 pixels adjacent to the wall edge. Figure 12 shows results of the velocity gradient with 3,4 , and 5 pixels adjacent to the wall edge (denoted 3-, 4-, and 5-pixel, respectively, to include the pixel representing the wall edge) at mean volume flow rates of 50, 100, 150, and $200 \mathrm{~mL} / \mathrm{min}$. The velocity gradient of the theoretical parabolic velocity profile at the wall edge was also listed. The velocity gradient rose from 32.7 to 131 seconds $^{-1}$ with an increase in the mean volume flow rate from 50 to $200 \mathrm{~mL} / \mathrm{min}$. The percentage of error relative to the theoretical value was calculated.

The decorrelation-based flow velocity measurement had good estimation of the velocity gradient using the 3 pixels. The error values were $3 \%, 14 \%, 7 \%$, and $21 \%$ for the mean flow rates of 50,100,150, and $200 \mathrm{~mL} / \mathrm{min}$, respectively. The lateral speckle tracking required more pixels for more accurate estimation of the velocity gradient. The 5pixel had the best estimation with 32\%,38\%, $14 \%$, and 39\% error for the mean flow rates of 50,100, 150, and 200 $\mathrm{mL} / \mathrm{min}$, respectively. This level of error was higher than that in the decorrelation-based flow velocity measurement. The trend of high error in velocity gradient estimation at a high volume flow rate was the same as in the decorrelationbased flow velocity measurement, which can be observed in Figure 12. At $200 \mathrm{~mL} / \mathrm{min}$, both decorrelation-based and lateral spectral-tracking flow velocity measurement methods were less accurate for prediction of the rapid rise in the flow velocity at the wall edge.

\section{Discussion}

The decorrelation flow velocity measurement method was shown to be more accurate than the lateral speckletracking method for prediction of the velocity gradient at 
the wall edge. Although the decorrelation-based method has a limitation on measuring the high flow velocity, as evident in Figure 7, it is more suitable for predicting the wall edge velocity because the decorrelation method uses a smaller spatial window and also performs the decorrelation over frames, whereas the other techniques just do correlations over space. The maximum velocity gradient error was $21 \%$ at the mean volume flow rate of $200 \mathrm{~mL} / \mathrm{min}$, as shown in Figure 12. Figure 13 shows the theoretical velocity versus decorrelation-based flow velocity. The underestimation of flow velocity increased at the high volume flow rate. In this study, the limit for accurate decorrelation measurement of flow velocity was about $100 \mathrm{~mm} / \mathrm{s}$, which was determined by the beam correlation width $(\approx 0.3 \mathrm{~mm})$ and the acoustic frame rate $(348 \mathrm{~Hz})$. This upper limit of the flow velocity was higher than the measured maximum flow velocity $(\approx 80$ $\mathrm{mm} / \mathrm{s}$ ) because the effective beam width in tissue is larger than the beam width in blood-mimicking fluid. The tissue speckles provide a stronger reflection compared to speckles in fluid, which means that the reflected RF signal would appear to attenuate faster across the beam in fluid than in tissue. This difference in the effective beam width may cause the upper limit of the flow velocity change since we calibrated the motion versus decorrelation on a tissue phantom and then used it to measure the scatterer motion in fluid.

The lateral speckle-tracking flow velocity measurement method could measure the whole velocity profile (Figure 7). This function is shown in Figure 14 on the theoretical velocity versus lateral speckle-tracking flow velocity. Due to the correlation window spatial smoothing, the accuracy for predicting the edge velocity gradient was limited. The most accurate prediction of the velocity gradient was only $14 \%$ at the mean volume flow rate of 150 $\mathrm{mL} / \mathrm{min}$, as shown in Figure 12.

Figure 11. A, B-mode image of the longitudinal view of the lumen with the fluid mixture flowing at the mean volume flow rate of $150 \mathrm{~mL} / \mathrm{min}$. B, Closeup views of pixels in the lumen and near the top and bottom edges as well as the $b-b$ line.

A

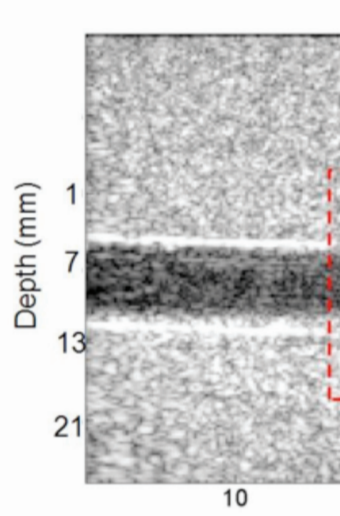

B

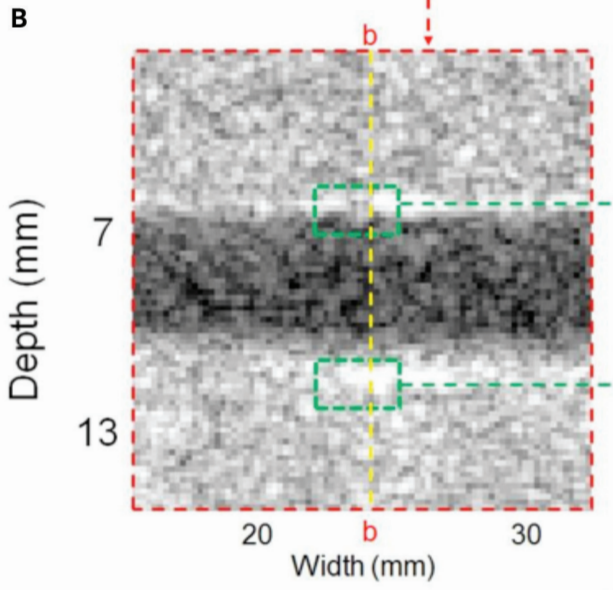

b
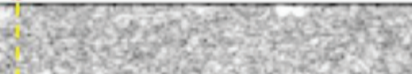

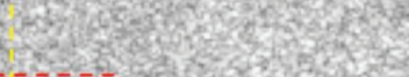
- $-2-10$ a

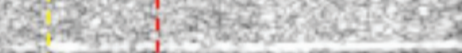
syition iston When

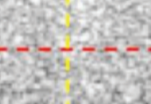
20 Width $(\mathrm{mm})$ Width $(\mathrm{mm})$ 
The advantage of speckle decorrelation-based flow velocity measurement is that blood velocity profiles can be determined across a vessel; thus, entire blood velocity profiles can be obtained along the vessel. In addition, the speckle decorrelation-based flow velocity measurement has higher spatial resolution than Doppler ultrasound measurements and can measure blood velocities near a vessel wall, accurately measuring the wall shear.
We observed that the top layer had a small beam correlation width in the near field. This small beam correlation width is due to the inhomogeneous beam profile in the extreme near field, and this phenomenon was proposed by Li et al. ${ }^{17}$ When scatterers move across the near field, rapid modulation of signal amplitudes may occur due to phase fluctuation in the near field, and speckle decorrelation occurs at a faster rate than in other regions. This fast

Figure 12. Flow velocity gradient at the wall edge at mean volume flow rates of 50,100, 150, and $200 \mathrm{~mL} / \mathrm{min}$.

\begin{tabular}{|c|c|c|c|c|c|}
\hline \multirow[b]{2}{*}{ Mean volume flow rate, $\mathrm{mL} / \mathrm{min}$} & \multicolumn{5}{|c|}{ Velocity Gradient, $\mathrm{s}^{-1}$ (\% Error) } \\
\hline & & 50 & 100 & 150 & 200 \\
\hline Theoretical (parabolic) velocity profile (red lines) & & 32.7 & 65.3 & 98.0 & 131 \\
\hline Decorrelation-based velocity profile (green lines with squares) & $\begin{array}{l}\text { 3-pixel } \\
\text { 4-pixel } \\
\text { 5-pixel }\end{array}$ & $\begin{array}{l}33.7(3) \\
27.2(17) \\
29.7(9)\end{array}$ & $\begin{array}{l}56.2(14) \\
63.4(3) \\
75.6(16)\end{array}$ & $\begin{array}{c}91.4(7) \\
108.0(10) \\
93.5(4.6)\end{array}$ & $\begin{array}{r}104.0(21) \\
98.9(25) \\
89.7(32)\end{array}$ \\
\hline Lateral speckle-tracking velocity profile (blue lines with circles) & $\begin{array}{l}\text { 3-pixel } \\
\text { 4-pixel } \\
\text { 5-pixel }\end{array}$ & $\begin{array}{l}19.0(42) \\
21.0(36) \\
22.3(32)\end{array}$ & $\begin{array}{l}30.9(53) \\
36.8(44) \\
40.7(38)\end{array}$ & $\begin{array}{l}77.5(21) \\
81.4(17) \\
84.1(14)\end{array}$ & $\begin{array}{l}64.0(51) \\
73.4(44) \\
79.7(39)\end{array}$ \\
\hline
\end{tabular}
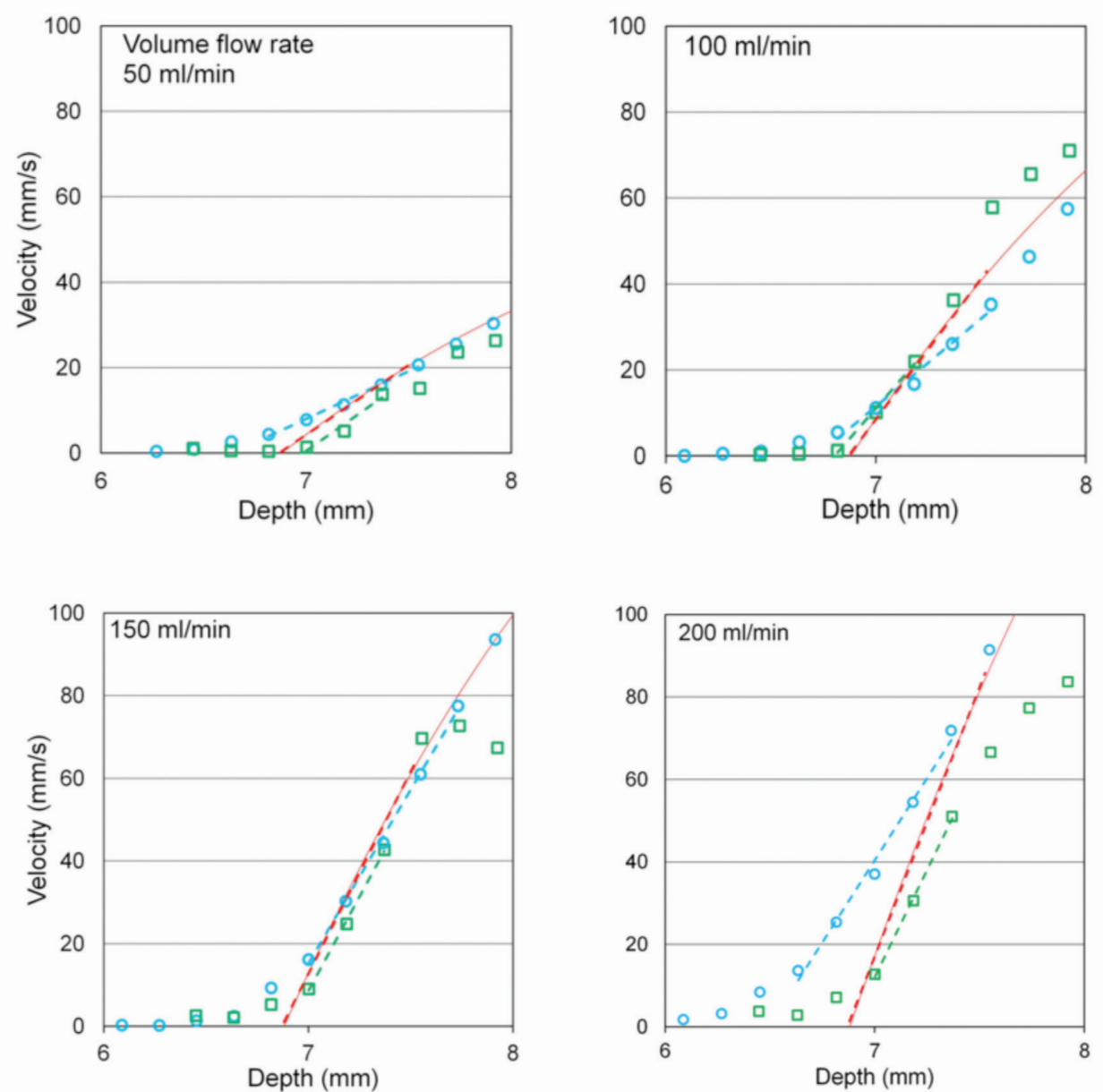


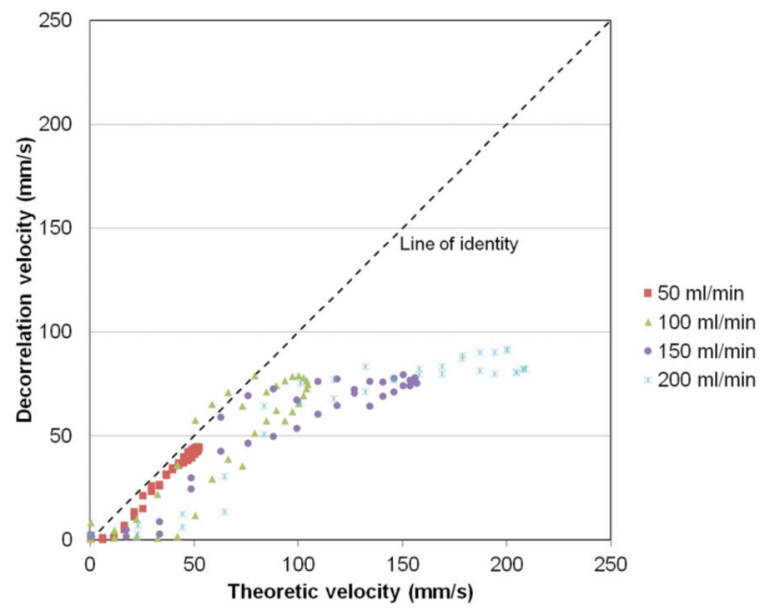

Figure 13. Theoretical velocity versus decorrelation-based flow velocity.

decorrelation results in a reduction in the mean beam correlation width in the near field, as shown in Figure 4. In addition, the outliers were observed especially in the near (layer 1) and far (layer 7) fields. The near outlier (layer 1) was caused by the inhomogeneous beam profile, and the deeper outlier (layer 7) was due to low signal levels caused by attenuation in the tissue-mimicking phantom. We performed only 4 measurements, and additional measurements would be ideal to obtain a more robust model of the transducer beam.

Figure 14. Theoretical velocity versus correlation-based lateral speckletracking velocity.

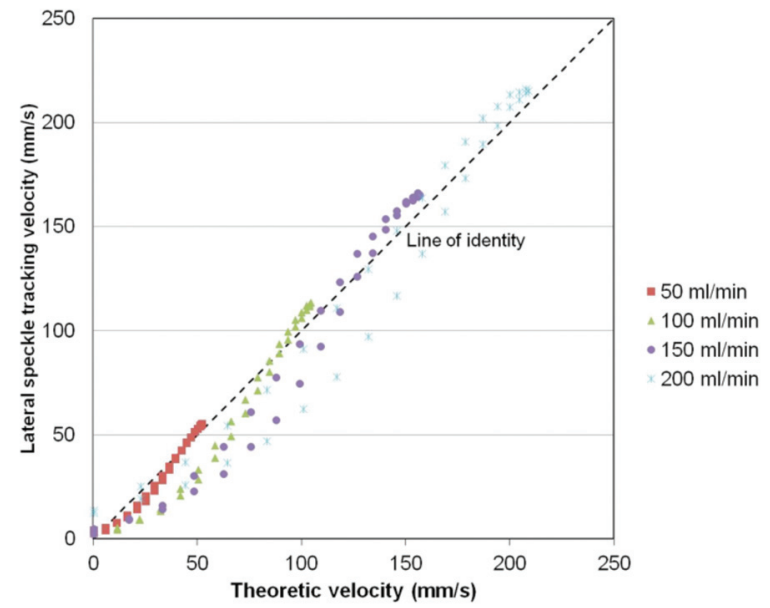

An additional observation is that a different number of pixels was necessary to determine accurate velocity gradients from the transverse and longitudinal flow velocity profiles. This trend is not general because the number of pixels for velocity gradient estimation depends on the size of the correlation window, which causes a smoothing effect on the edges, for lateral speckle tracking.

In this study, 4 pixels were selected in a transition region between the phantom and fluid in a B-mode image to determine the edges using the second-order gradient method. We used a 9-MHz transducer, which has a pixel resolution of about $0.1 \mathrm{~mm}$, and identified 4 pixels in the transition region that were brighter. Therefore, 4 pixels were selected between the phantom and fluid in a B-mode image to determine the edges using the second-order gradient method. Thus, to use this approach, the B-mode spatial resolution needs to be high enough to detect multiple pixels in the transition region. In addition, the contribution of errors affecting edge detection due to variations in speed of sound estimates needs to be considered for translation of this edge detection method to in vivo studies because the differences in sound speed will distort the position of the edges and the shape of the speckles and also affect the beam focusing.

One substantial limitation of the speckle decorrelationbased flow velocity measurement is that time averaging of the correlation coefficient is necessary to reduce the noise from large transient signals such as tissue and pulsatile motion. In this study, the flow velocity profiles were obtained by time averaging 700 frames of the correlation coefficient using the speckle decorrelation method. Since the pulsatile flow and wall motions occur in vessels, this time averaging of the correlation coefficient needs to be reduced to detect the flow velocity changes and vascular wall motions. We achieved the measurement of blood velocity gradients and vascular wall motions in a vessel during cardiac cycles by using the decorrelation measurements with short time averaging. ${ }^{33}$

A second limitation of the speckle decorrelation method is that the accuracy of velocity gradient measurement is dependent on the direction of the flow velocity measurement. In our study, the velocity gradients were measured from vertical (axial directions) lines in the lumen because the vertical lines had higher spatial resolution and showed more accurate edge detection than horizontal (lateral directions) lines. However, the blood velocity profile is not symmetric in a vesse ${ }^{1}$; thus, the velocity gradient needs to be obtained along the entire vessel wall. This limitation needs to be considered for translation of this decorrelation-based flow velocity measurement method into clinical studies. 
In addition to these limitations, we also encountered a substantial estimated error for measurement of the velocity gradient at walls under a high volume flow rate. The rapid rise in the flow velocity near the wall may contribute to this high error in velocity gradient measurements. This limitation can be overcome by using an ultrasound machine with a very high frame rate. Currently, ultrasound machines can produce very high frame rates by transmitting an unfocused plane wave and then focusing the receive waves. ${ }^{34}$ The rapid rise in decorrelation near the wall due to the high flow rate can be measured by using a transducer with a very high frame rate.

In summary, the decorrelation flow velocity measurement method not only can predict the edge velocity gradient more accurately than the lateral speckle-tracking flow velocity measurement method but also has the advantage of the transverse view of the vessel for in vivo patient studies. A long section of a vessel with a uniform size is difficult to identify in a patient because of the tortuosity and branching of vessels. Additional laboratory study is planned using particle imaging velocimetric methods ${ }^{35}$ to establish the validity of these methods in variable vascular geometries, and ongoing clinical work is being performed to validate the clinical utility of these methods in vivo. ${ }^{33}$

An application and future study of the decorrelation flow velocity measurement method is the measurement of vascular wall shear stress to assess the risk of vascular and heart disease. This decorrelation-based velocity measurement method may be very helpful for determining the flow velocity gradient and the vascular wall shear stress in healthy and high-risk patient populations to noninvasively study and assist in the diagnosis of cardiovascular disease.

\section{References}

1. Ku DN. Blood flow in arteries. Annu Rev Fluid Mech 1997; 29:399-434.

2. Kroll MH,Hellums JD, Mcintire LV, Schafer AI, MoakeJL. Platelets and shear stress. Blood 1996; 88:1525-1541.

3. Vennemann P, Lindken R, Westerweel J. In vivo whole-field blood velocity measurement techniques. Exp Fluids 2007; 42:495-511.

4. Gill RW.Measurement of blood flow by ultrasound: accuracy and sources of error. Ultrasound Med Biol 1985; 11:625- 641.

5. Brands PJ, Hoeks APG, Hofstra L, Reneman RS. A noninvasive method to estimate wall shear rate using ultrasound. Ultrasound Med Biol 1995; 21:171-185.

6. Samijo SK, Willigers JM, Barkhuysen R, et al. Wall shear stress in the common carotid artery as function of age and gender. Cardiovasc Res 1998; 39:515-522

7. Bambi G, Morganti T, Ricci S, et al. A novel ultrasound instrument for investigation of arterial mechanics. Ultrasonics 2004; 42:731-737.
8. Tortoli P, Morganti T, Bambi G, Palombo C, Ramnarine KV. Noninvasive simultaneous assessment of wall shear rate and wall distention in carotid arteries. Ultrasound Med Biol 2006; 32:1661-1670.

9. Trahey GE, Allison JW, von Ramm OT. Angle independent ultrasonic detection of blood flow. IEEE Trans Biomed Eng 1987; 34:965-967.

10. Bohs LN, Friemel BH, Trahey GE. Experimental velocity profiles and volumetric flow via two-dimensional speckle tracking. Ultrasound Med Biol 1995; 21:885-898.

11. Bohs LN, Geiman BJ, Anderson ME, Breit SM, Trahey GE. Ensemble tracking for $2 \mathrm{D}$ vector velocity measurement: experimental and initial clinical results. IEEE Trans Ultrason Ferroelectr Freq Control 1998; 45:912924.

12. Gallippi CM, Bohs LN, Aderson ME, Congdon AN, Trahey GE. Lateral blood velocity measurement in the carotid artery via speckle tracking. In: Proceedings of the 2001 IEEE Ultrasonics Symposium. Piscataway, NJ: Institute of Electrical and Electronics Engineers; 2001:1451-1455.

13. Aoudi W, Liebgott H, Needles A, Yang V, Foster FS, Vray D. Estimation methods for flow imaging with high frequency ultrasound. Ultrasonics 2006; 44(suppl 1):e135-e140.

14. Li W, van der Steen AFW, Lancée CT, Gussenhoven EJ, Nicolaas B. Temporal correlation of blood scattering signals in vivo from radiofrequency intravascular ultrasound. Ultrasound Med Biol 1996; 22:583-590

15. Li W, van der Steen AFW, Lancée CT, Céspedes I, Nicolaas B. Blood flow imaging and volume flow quantitation with intravascular ultrasound. Ultrasound Med Biol 1998; 24:203-214.

16. Bamber J, Hasan P, Cook-Martin G, Bush N. Parametric imaging of tissue shear and flow using B-scan decorrelation rate [abstract]. JUltrasound Med 1988; 7 (suppl):S135.

17. LiW,Lancée CT, Céspedes EI, van der Steen AF, Bom N. Decorrelation of intravascular echo signals: potentials for blood velocity estimation. J Acoust Soc Am 1997; 102:3785-3794

18. Chen JF, Fowlkes JB, Carson PL, Rubin JM, Adler RS. Autocorrelation of integrated power Doppler signals and its application. Ultrasound Med Biol 1996; 22:1053-1057.

19. Tuthill TA, Krücker JF, Fowlkes JB, Carson PL. Automated threedimensional US frame positioning computed from elevational speckle decorrelation. Radiology 1998; 209:575-582.

20. Fowlkes JB, Carson PL, Moskalik A, Chen JF, Rubin JM, inventors Method and apparatus for composition and display of three-dimensional image from two-dimensional ultrasound scan data. US Patent 6,059,727. 2000 .

21. Rubin JM, Fowlkes JB, Tuthill TA, et al. Speckle decorrelation flow measurement with B-mode US of contrast agent flow in a phantom and in rabbit kidney. Radiology 1999; 213:429-437.

22. Rubin JM, Tuthill TA, Fowlkes JB. Volume flow measurement using Doppler and grey-scale decorrelation. Ultrasound Med Biol 2001;27:101109.

23. Papoulis A. Probability, Random Variables, and Stochastic Processes. New York: McGraw-Hill; 1965. 
24. Carson PL, Johnson ML, Holmes JH. Image quality and practicality of scanning large abdomens with large, low frequency and smaller, high frequency transducers. In: White D, Lyons EA (eds). Ultrasound in Medicine. Vol 4. New York, NY: Plenum Press; 1978:161-162.

25. Banjavic RA, ZagzebskiJA, Madsen EL, Jutila RE. Ultrasound beam sensitivity profile changes in mammalian tissue. In: White D, Lyons EA (eds). Ultrasound in Medicine. Vol 4. New York, NY: Plenum Press 1978:515518.

26. Adler RS, Rubin JM, Fowlkes JB, Carson PL, Pallister JE. Ultrasonic estimation of tissue perfusion: a stochastic approach. Ultrasound Med Biol $1995 ; 21: 493-500$.

27. Oosterveld BJ, Thijssen JM,VerhoefWA. Texture of B-mode echograms: 3-D simulations and experiments of the effects of diffraction and scatterer density. Ultrasound Imaging 1985; 7:142-160.

28. Widmaier EP, RaffH, Strang KT. Vander's Human Physiology. New York, NY: McGraw Hill; 2006.

29. Othman NS, Jaafar MS, Rahman AA, Othman ES, Rozlan AA. Ultrasound speed of polymer gel mimicked human soft tissue within three weeks. Int J Biosci Biochem Bioinform 2011; 1:223-225.

30. Shung KK. Diagnostic Ultrasound: Imaging and Blood Flow Measurements. Boca Raton, FL: CRC Press; 2006.

31. Moshfeghi M, Waag RC. In vivo and in vitro ultrasound beam distortion measurements of a large aperture and a conventional aperture focussed transducer. Ultrasound Med Biol 1988; 14:415-428.

32. Lubinski MA, Emelianov SY, O’Donnell M. Speckle tracking methods for ultrasonic elasticity imaging using short-time correlation. IEEE Trans Ultrason Ferroelectr Freq Control 1999; 46:82-96.

33. Park DW, Kruger GH, Rubin JM, et al. In vivo vascular wall shear rate and circumferential strain of renal disease patients. Ultrasound Med Biol 2012; 39:241-252

34. Zhao H, Song P, Urban MW, Greenleaf JF, Chen S. Shear wave speed measurement using an unfocused ultrasound beam. Ultrasound Med Biol 2012; 38:1646-1655.

35. Adrian RJ, Westerweel J. Particle Imaging Velocimetry. Cambridge, England: Cambridge University Press; 2010. 